\title{
2 Jacobis Entwurf der realen Transgressivität
}

Friedrich Heinrich Jacobis philosophisches Projekt steht und fällt mit der kantkritischen Einsicht, dass in der sinnlichen Empfindung der Außenwelt eine Gewissheit vorliegt, deren epistemischer Status unbezweifelbar ist und deren Inhalt notwendig die bloße Form von Repräsentationalität (Vorstellung) übersteigt: „Dinge überhaupt“ sind dem Subjekt als reale, d.h. a) in ihrem Dass-Sein und SoSein nicht vollständig vom Subjekt konstituierte und b) als an sich selbst wirklich bestehende, immer schon gegeben. Jacobi bezeichnet diese Position als „Realismus“201 und sich selbst als „Realisten“202: „[...] weil mir Dinge gegeben seyn müssen, ehe ich Verhältnisse einzusehen im Stande bin.“203 Zugleich bleibt Jacobi jedoch nicht bei dem bloßen, unbegründbaren wie unentfaltbaren Faktum einer solchen Gewissheit stehen. Vielmehr müssen für ihn in dieser Gewissheit, wenn sie Anspruch auf den Status des „Wissens“ erheben will, sowohl das evident Gegebene (das Wirkliche) selbst bestimmt sein, ohne auf die bloße Bestimmbarkeit durch das Subjekt zurückzufallen (Kant), als auch die eigenen subjektiven Bedingungen des Gegebenseins von Realität bestimmbar sein. Die unbezweifelbar gegebene Gewissheit des Realen muss folglich durch sich selbst so zu verstehen sein, dass in ihr die Formen, welche das Reale jenseits des bloßen Subjektiven an sich selbst haben soll, zum Vorschein kommen, und es muss zu begründen sein, warum diese Formen als nicht bloß subjektive zugleich in der Form subjektiver Gewissheit erscheinen. Das Ziel des folgenden Kapitels ist es, diesen einen Zusammenhang jenseits der zahlreichen Diskussionslinien der jacobischen Philosophie und abseits der Problemlinien der Sekundärliteratur, rein textimmanent, in aller Kürze herauszuarbeiten, um zu zeigen, inwiefern Jacobi den Schritt über die „Kantische Grenzlinie“ (so Hölderlin in einem Brief an Neuffer vom 10. Oktober 1794) wagt und welche Probleme sich aus seinem Ansatz ergeben.

Ausgehend von der bereits skizzierten Fundamentalkritik an der systemsprengenden Widersprüchlichkeit der Architektur des „Ding an sich“ (vgl. Kap. 1.1.6), welche für Jacobi die Unhaltbarkeit eines auf bloße Erscheinung reduzierten Realen für das Subjekt erweist und weit über eine Kritik an der bloßen Überdehnung der Kausalitätskategorie hinausgeht, führt Jacobi die Begriffe des "Glaubens" und der „Offenbarung“ in die theoretische Philosophie ein, um die Leerstelle des „Ding an sich“ zu füllen und seine Realform dem Subjekt zugäng-

201 Jacobi: David Hume, JWA 2,1, S. 32.

202 Jacobi: David Hume, JWA 2,1, S. 10.

203 Jacobi: David Hume, JWA 2,1, S. 10.

Ә OpenAccess. () 2021 Claudia Wirsing, publiziert von De Gruyter. (cc)BY Dieses Werk ist lizenziert unter einer Creative Commons Namensnennung 4.0 International Lizenz. 
lich zu machen. Beide Begriffe werden eng mit dem Konzept der „Vernunft“ verknüpft, das Jacobi, funktional weitaus deutlicher als Kant, in kategorialer Differenz vom „Verstand“ unterscheidet. „Glaube“ - den Jacobi vom „blinden Glauben“204 absetzt und für den er auf eine Hume’sche Begriffsverwendung zurückgreift ${ }^{205}$ - meint bei ihm „eine stärkere, lebendigere, mächtigere, festere, anhaltendere Vorstellung eines Gegenstandes, als die Einbildungskraft allein je $\mathrm{zu}$ erreichen im Stande ist. “206 Deshalb kann der epistemische Glaube nicht „blind“ sein, weil ihm Gründe eigener Art für das Geglaubte zur Verfügung stehen: nämlich die perennierende Intensität des Glaubens selbst. Der Vorgang des Glaubens fungiert so epistemisch als Beglaubigung der Wirklichkeit, d.h. der unabhängigen bestimmten Existenz des Glaubensgegenstandes. Dieses im epistemischen „Glauben“ sich ereignishaft vollziehende und unmittelbare, d.h. nicht erst durch Bewusstseinsleistungen konstituierte, sondern vielmehr in diese von außen eindringende Sichselbstgeben der Dinge in ihrer transsubjektiven Realität, nennt Jacobi „Offenbarung“207, durch welche die epistemische Norm der Wahrheit einer Vorstellung erst ihren letzten Grund erhält: „Wahrheit ist Klarheit, und bezieht sich überall auf Würklichkeit, auf Facta. “208 Gemeinsam mit der subjektiven Vollzugsform der sinnlichen Empfindung konturieren „Glaube“ und „Offenbarung“ das Seelenvermögen der „Vernunft“, die für Jacobi nichts anderes als „der Charakter seiner [des Menschen, C.W.] besonderen Sinnlichkeit sey“209: als besondere Sinnlichkeit, die als „Organ der Vernehmung des Uebersinnlichen“210 in den subjektiven Sinnesdaten über diese hinausgreift. Diese Dialektik von Sinnlichkeit und Über-Sinnlichkeit, d.h. von einer gesteigerten sinnlichen Eindrücklichkeit, die in der besonderen Intensität ihrer Ausführung über sich selbst auf ein Außer-Sinnliches hinausgreift und dessen Dass-Sein beglaubigt (des „Uebersinnlichen, wahrhaft-Realen“211, entwickelt Kants Vernunftbegriff in entscheidender Weise weiter. Wie bei Kant sind die Gegenstände der Vernunft letztlich nur zu „glauben“: Zugleich aber wird dieser Glaube als höhere Art des Wissens entworfen und dem Verstandeswissen mit seiner begrifflichen Form übergeordnet. „Übersinnlich“ ist dabei in doppelter, einander entgegengesetzter Richtung gemeint: den Raum des deutlichen Bewusstseins nach ,unten', in

204 Jacobi: David Hume, JWA 2,1, S. 18.

205 Vgl. Jacobi: David Hume, JWA 2,1, S. 24.

206 Jacobi: David Hume, JWA 2,1, S. 29.

207 Jacobi: David Hume, JWA 2,1, S. 32 f.

208 Jacobi: Über die Lehre des Spinoza, S. 129.

209 Jacobi: David Hume, JWA 2,1, S. 66.

210 Jacobi: Einleitung, JWA 2,1, S. 377.

211 Jacobi: Einleitung, JWA 2,1, S. 383. 
Richtung der empirischen Realität materieller Dinge, wie auch nach ,oben', in Richtung der Gewissheit des wirkenden Vorhandenseins letzter Ideen wie Freiheit und Gott, transzendierend. Die Vernunft im Menschen setzt beides immer schon voraus und beglaubigt es zugleich im Gefühl unabweisbarer Evidenz ihres tatsächlichen Vorhandenseins. ${ }^{212}$ Damit gewinnt Jacobi den Begriff eines Erkenntnisvermögens, das sich wiederum dem Theoriegefüge der ,intellektuellen Anschauung“ (Fichte, Schelling, Hölderlin), der „scientia intuitiva“ bzw. dem „intellectus archetypus“ (Kant) um 1800 einfügt und eine weitere Alternative zur bloß begrifflichen Erkenntnis darstellen soll. Die Vernunft als Vermögen, das Wahre der einzelnen empirischen Gegenstände unabhängig von uns und auch das Wahre des unendlichen Grundes in Gott als immer schon gegenwärtig und erkannt vorauszusetzen, opponiert deutlich gegen das romantische „Streben nach dem Unendlichen“ als einem in „unendlicher Annäherung“ sich Befindenden:

Ich berufe mich auf ein unabweisbares unüberwindliches Gefühl als ersten und unmittelbaren Grund aller Philosophie und Religion; auf ein Gefühl, welches den Menschen gewahren und inne werden läßt: er habe einen Sinn für das Übersinnliche. Diesen Sinn nenne ich Vernunft, zum Unterschiede von den Sinnen für die sichtbare Welt. ${ }^{213}$

Mit dem Entwurf dieses Vermögens koppelt Jacobi die Gewissheit des Endlichen und des Unendlichen, d.h. des Dass-Seins und des So-Seins einer außersubjektiven Realität und die Evidenz der Gotteserfahrung strukturell aneinander, um sie sich gegenseitig abstützen zu lassen. Demgemäß unterscheidet Jacobi den Modus von „Sein überhaupt“, den die Vernunft gewährt, von dem apriorischen Modus von Seiendheit, der den kategorialen Verstandesbestimmungen zugrunde liegt:

Das Ist des überall nur reflectierenden Verstandes ist überall auch nur ein relatives Ist, und sagt mehr nicht aus, als das bloße einem Andern gleich seyn im Begriffe; nicht das substanzielle Ist oder Seyn. Dieses, das reale Seyn, das Seyn schlechthin, giebt sich im Gefühle allein zu erkennen; in demselben offenbart sich der gewisse Geist. ${ }^{214}$

Das „oberherrliche Wissen“215 der Vernunft macht demnach eine Sache an sich selbst, als nur mit sich selbst Gleiche in der individuellen Substanz ihres Seins, zugänglich, wohingegen die Konstruktionen des Verstandes in Begriffen unterschiedliche Vorstellungen in Relation zueinander, also rein bewusstseinsintern, auf Identität prüfen. Indem Jacobi also im komplexen Gesamtvermögen der Ver-

212 Vgl. Jacobi: Einleitung, JWA 2,1, S. 378.

213 Jacobi: Über die Lehre des Spinoza, S. 306 [Vorbericht, 1819].

214 Jacobi: Einleitung, JWA 2,1, S. 424.

215 Jacobi: Einleitung, JWA 2,1, S. 424. 
nunft Kants Architektur der begrifflichen Erkenntnisvermögen wesentlich umorganisiert und erweitert, schafft er die Grundlage dafür, die bei Kant angedachte und doch systematisch verhinderte Transgressivität des reinen Begriffs der Realität, der sich als Notwendigkeit aus den Widersprüchen des „Ding an sich“ ergeben hatte, überhaupt denken zu können. Zugleich sichert Jacobi - darin der Grundsatzfrage der Klassischen Deutschen Philosophie verhaftet - über die nicht weiter abzuleitende, unmittelbare, sich im Gefühl der Gewissheit selbstbezeugende Letzteinsicht der Vernunft in das Dass-Sein und das So-Sein von „Realität überhaupt“, von menschlicher Freiheit und von der Persönlichkeit Gottes, dem mittelbaren, stets relativen, begriffsabhängigen und $\mathrm{zu}$ bezweifelnden propositionalen Wissen mitsamt seiner Normen ein Fundament $\mathrm{zu}$, das freilich nicht mehr sinnvoll innerhalb der Maßstäbe und Verhandlungsformen begrifflicher Erkenntnis zu erfassen ist. ${ }^{216}$ In der Gewissheit der Vernunft offenbaren sich dem Menschen die letzten Gründe und Zusammenhänge der „Wirklichkeit überhaupt“ als ihm grundsätzlich und vorgängig aufgeschlossen. Diese, alles Erfahren und alles Wissen durchwirkende und beherrschende Erschlossenheit kann jedoch dem Menschen nicht anders denn als grundlose, dem Subjekt vorgängig ebenso gegebene wie entzogene Dimension der „Lichtung des Seins“ (Heidegger) zukommen: „[...] daß es ein Wissen aus der ersten Hand gebe, welches alles Wissen aus der zweyten (die Wissenschaft) erst bedinge, ein Wissen ohne Beweise, welches dem Wissen aus Beweisen nothwendig vorausgehe, es begründe, es fortwährend und durchaus beherrsche.“217 In der Spinoza-Schrift heißt es noch deutlicher:

Wie können wir nach Gewißheit streben, wenn uns Gewißheit nicht zum voraus schon bekannt ist [...]? Dieses führt uns zu dem Begriffe einer unmittelbaren Gewißheit, welche nicht allein keiner Gründe bedarf, sondern schlechterdings alle Gründe ausschließt, und einzig und allein die mit dem vorgestellten Dinge übereinstimmende Vorstellung selbst ist. Die Überzeugung aus Gründen ist eine Gewißheit aus der zweiten Hand. ${ }^{218}$

Indem Jacobi den Seinsbegriff Spinozas als „absolute Position“ mit seiner Idee der Vernunft als unmittelbar selbstgewissem Vermögen der Erschlossenheit des

216 Vgl. dazu Jacobi: David Hume, JWA 2,1, S. 24, 38; Jacobi: Einleitung, JWA 2,1, S. 375f., 402f., 427; Jacobi: Über die Lehre des Spinoza, S. 65, 113f., 310 f.

217 Jacobi: Einleitung, JWA 2,1, S. 375.

218 Jacobi: Über die Lehre des Spinoza, S. 113. Diese Kritik der Idee einer grundlosen epistemischen Verlässlichkeit bzw. der Selbstwidersprüchlichkeit eines Begriffs der Fundierung inferenziellen Wissens in einem unmittelbaren Wahrnehmungswissen findet sich auch bei Quine, Sellars, Davidson und Brandom. 
Realen verbindet, nimmt er dergestalt bereits in Ansätzen die hermeneutische Ontologie Heideggers und Gadamers vorweg.

Ein wesentlicher Zug dieser Umorganisation der Vermögenslehre von Kant zu Jacobi besteht darin, die bereits von Kant von einer vertikalen (obere - untere) auf eine horizontale Struktur umgestellte Ordnung der Seelenvermögen des Menschen weiter zu dezentrieren und ihr latent hierarchisches Schema aufzulösen.

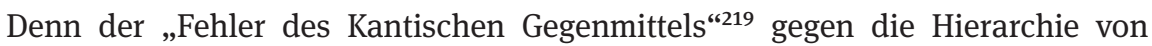
„oberen“ und „unteren“ Erkenntnisvermögen liegt nach Jacobi darin, in der horizontalen, gleichgewichtigen Beiordnung von Sinnlichkeit und Verstand als gleichermaßen notwendigen Bestandteilen des Erkennens zugleich den eigenen Erkenntnis- und Wissenscharakter sinnlicher Empfindung gänzlich abgeschafft zu haben: Kant „reiniget die Sinnlichkeit in solchem Maaße, daß sie, nach dieser Reinigung, die Eigenschaft eines Wahrnehmungsvermögens ganz verliert. Wir erfahren, daß wir durch die Sinne überall nichts Wahres erfahren“220 . Gegen Kant setzt Jacobi die unhintergehbare, selbstevidente und deshalb für ihn unbestreitbare „Voraussetzung, daß Wahrnehmung, im strengsten Wortverstande - sey, und daß ihre Wirklichkeit und Wahrhaftigkeit obgleich ein unbegreifliches Wunder, dennoch schlechthin angenommen werden müsse“221 . Jacobi gesteht also nicht nur der sinnlichen Wahrnehmung eine eigene, in sich bereits abgeschlossene und auf den Verstand irreduzible Erkenntnisfähigkeit zu, die das kantische Schema durchbricht, nach welchem die Sinnesdaten für sich gar nichts bedeuten, solange sie nicht durch die Anschauungsformen und die reinen Begriffe bzw. die Schemata des Verstandes interpretiert worden sind: „Jede Wahrnehmung ist folglich an sich schon ein Begriff.“222 Er ordnet diese Erkenntnisfähigkeit überdies in aristotelischer Tradition dem Vermögen der Vernunft $\mathrm{zu}$, wodurch das ehemals „unterste“ und das ehemals „oberste“ Vermögen, sinnliche Wahrnehmung und Vernunft, zusammenfallen und sich gemeinsam vom Verstand als Vermögen deutlicher Begriffe unterscheiden.

Der damit verbundene unmittelbare Wissenscharakter der sinnlichen Wahrnehmung wird nun von Jacobi weiter zu entwickeln gesucht, auch um seine innere Bestimmtheit gegen den Vorwurf bloß dunklen Anwesenheitsgefühls sichtbar zu machen. Vor allem im Hume-Dialog unternimmt er es folglich, die in der sinnlichen Wahrnehmung evident gegebene reine Form des Realseins der äußeren Dinge einer Analyse zu unterziehen: „Sie werden eine Empfindung gewahr, und in dieser Empfindung eine andere Empfindung, durch die Sie empfinden, daß diese

219 Jacobi: Einleitung, JWA 2,1, S. 382.

220 Jacobi: Einleitung, JWA 2,1, S. 382; vgl. auch Jacobi: Einleitung, JWA 2,1, S. 389.

221 Jacobi: Einleitung, JWA 2,1, S. 390.

222 Jacobi: David Hume, JWA 2,1, S. 86. 
Empfindung die Ursache von jener Empfindung ist“223. Die Empfindung des Realseins der äußeren Dinge ist eine reflexive: In ihr ist das Empfundene als Ursache der Empfindung, d.h. das intentionale Objekt der Empfindung, intern differenziert vom intentionalen Akt ihres subjektiven Gewahrwerdens. Das „würklich äusserliche, an sich vorhandene Wesen“224 der „Dinge an sich“ teilt sich in der Empfindung als Anderes zur Empfindung als ihr Grund mit. Die Empfindung des Realen selbst besteht darin, eine Evidenzerfahrung („Glauben“) als Empfindung zu erlangen, die zugleich ihren intentionalen Gehalt als ein Anderes zur Form der Empfindung dokumentiert: nämlich als außer der Empfindung wirklich vorhandenes Etwas-als-Anderes-zum-Subjekt. Im gewissen subjektiven Empfinden des Realseins der Dinge differenziert sich in der Form des Empfindens ein Raum aus, der als Drittes zum Unterschied des logischen Raums der Subjektivität und des logischen Raums der Realität figuriert ist: als Raum der Überschreitung des Subjektiven, der bis ins Subjekt hineinreicht und demgemäß sinnvoll nur als „Offenbarung“ bezeichnet werden kann, um nicht dem Setzungs- und Formgebungscharakter der transzendentalen Subjektivität zu unterstehen. Es ist die Beglaubigungskraft dieses „Gefühls“, in welchem sich die unmittelbare Empfindung unmittelbar äußert, welche Vorstellungen dort begleitet, wo ihr Gehalt als ein an sich wirklicher im Subjekt ankommt, und sie von bloßen „Erdichtungen“ unterscheidet. Gemäß Kant, dem Jacobi hier zweifelsohne folgt, ist diese Form der Realitätsgewissheit der intentionalen Objekte des Bewusstseins nicht als Eigenschaft der Vorstellungen selbst zu verstehen: ${ }^{225}$ Kein Inhalt kann als an sich notwendig wirklich erkannt werden, weil Realität gemäß der Widerlegung des ontologischen Gottesbeweises kein Prädikat erster Ordnung ist. Folglich kann der Index des Realseins als eigenständiges Gefühl die Vorstellungen des Bewusstseins nur begleiten: als durch die Natur selbst erregte, d.h. unwillkürliche Empfindung einer Anwesenheit, die dem deutlichen Vorstellungsinhalt in der nicht weiter abzuleitenden Realitätsgewissheit des Vorgestellten eine bestimmte Färbung gibt. Die von mir eingeführte Metapher der „Färbung“ scheint hier deshalb angebracht, weil Jacobi selbst den Einfluss des Realitätsgefühls auf die deutliche Vorstellung der Sache im Bewusstsein in einer bloß quantitativ-komparativen Nomenklatur zu beschreiben sucht: indem er nämlich diesen Glauben als ,eine stärkere, lebendigere, mächtigere, anhaltendere Vorstellung eines Gegenstandes, als die Einbildungskraft allein je zu erreichen im Stande ist“226, erfasst. Damit aber geht eben der irreduzible qualitative Aspekt, den das gewisse Realitätsgefühl

223 Jacobi: David Hume, JWA 2,1, S. 20.

224 Jacobi: David Hume, JWA 2,1, S. $20 \mathrm{f}$.

225 Vgl. Jacobi: David Hume, JWA 2,1, S. $28 \mathrm{f}$.

226 Jacobi: David Hume, JWA 2,1, S. 29. 
der subjektiven Vorstellung von außen einfügt, in der bloß internen Graduierung der Vorstellung verloren: Denn so lässt sich diese aufgrund der unbegrenzten Selbstaffektation des Denkens (gerade Wahnvorstellungen haftet die Eigenschaft an, stärker und mächtiger als alle Realvorstellungen zu sein) erneut nicht notwendig von bloßer „Erdichtung“ unterscheiden. An anderer Stelle zeigt Jacobi, dass er dies durchaus vor Augen hat: „[D]ie Vorstellungen können das Würkliche, als solches, nie darstellen. Sie enthalten nur Beschaffenheiten der würklichen Dinge, nicht das Würkliche selbst. “227 Hier wird das „Wirkliche selbst“ gerade als etwas begriffen, das zu der Form und dem Inhalt der Vorstellungen als Anderes, Irreduzibles und Qualitatives hinzutritt; das nicht innerhalb der Vorstellungen als deren quantitative Modifikation begriffen werden kann. Nur so bleibt es möglich, die „Ueberzeugung von dem eigenen Daseyn der Gegenstände unserer Vorstellungen“228 in skeptischer Hinsicht nicht als bloßen Schluss des Verstandes misszuverstehen. Dabei greift Jacobi erneut zu einem Wechselerweis selbstgewisser Empfindungen, um die Gewissheit, dass „das Reale dem Idealen, das Würkliche dem Möglichen, die Sache dem Begriff" 229 vorausgehe, d.h. dass die absolute Position des Seins der „Dinge an sich“ als jeder Vorstellung des Bewusstseins vorausgehend gedacht werden muss, evident $\mathrm{zu}$ machen.

Ich erfahre, daß ich bin, und daß etwas ausser mir ist, in demselben untheilbaren Augenblick; und in diesem Augenblicke leidet meine Seele vom Gegenstande nicht mehr als sie von sich selbst leidet. Keine Vorstellung, kein Schluß vermittelt diese zwiefache Offenbarung. ${ }^{230}$

Die Gewissheit des Realitätsgefühls der „Dinge an sich“ ist also nicht nur transzendent an der Gewissheit Gottes, sondern auch immanent am „Selbstgefühl“ des Ich geeicht; wir fühlen so unmittelbar die Realität der Dinge außer uns, wie wir uns selbst als anwesend und Gott als gewiss fühlen. In der wechselseitigen Beleuchtung bezeugen die unmittelbaren Empfindungen des Selbst, der Außenwelt und Gottes füreinander, dass ihre intentionalen Gehalte notwendig mehr sind als bloße Bewusstseinsform: dass in ihnen ein Anderes zum Subjekt in seinem Realsein in das Bewusstsein einfällt. Damit greift Jacobi Kants eigenes Argument aus der großen Anmerkung in der Vorrede B der Kritik der reinen Vernunft (KrV, B XL-XLI) bzw. aus dem in Auflage B hinzugefügten Abschnitt „Widerlegung des Idealism“ auf ${ }^{231}$, radikalisiert dieses aber zu einer realistischen Grundlegung

227 Jacobi: David Hume, JWA 2,1, S. 69.

228 Jacobi: David Hume, JWA 2,1, S. 36.

229 Jacobi: David Hume, JWA 2,1, S. 37.

230 Jacobi: David Hume, JWA 2,1, S. 37.

231 Vgl. Jacobi: David Hume, JWA 2,1, S. 86. 
der Vorstellungen des Bewusstseins im strukturanalogen Gefühl sich bedingender Offenbarungen der Gegenwärtigkeit von Ich, Welt und Gott.

Damit aber ist Jacobis Reflexion längst nicht erschöpft. Bisher sind erst der Realismus Jacobis und dessen systematische Verortung im Idealismus der Bewusstseinsformen entwickelt worden. Bezüglich unserer Fragestellung nach der bestimmten kategorialen Minimalstruktur des Realen setzt hier erst die eigentliche Überlegung über die ansichseiende, ,reine“ Form des Realen an, die sich aus der bloßen Dass-Gewissheit der ansichseienden Realität ableiten lassen soll. Eine wichtige Stellung nimmt dabei Jacobis Position zur ,objektiven Realität‘ der Kausalwirkung ein, die von Hume bezweifelt und demgemäß von Kant in das kategoriale Setting der transzendentalen Subjektivität eingegliedert worden war. Es ist Jacobis Ziel aufzuzeigen, wie es überhaupt dazu kommen konnte, dass das Kausalverhältnis nicht als etwas den Dingen in ihrer außersubjektiven Realität selbst Zukommendes und sie Bestimmendes, sondern als eine bloße Vorstellungsart des Subjekts, ob nun auf der Grundlage empirischer Daten gebildet (Hume) oder transzendental (Kant), missverstanden wurde: Ex negativo soll sich so die objektive Realität der Kausalität als ein wesentlicher Baustein der reinen Form des Realen erwiesen. Jacobis zentrales Argument ist dabei folgendes: „Diese [...] Täuschung wird [...] bewirkt, indem man den Begriff der Ursache mit dem Begriff des Grundes vermischt; jenem dadurch sein Eigentümliches entzieht, und ihn in der Spekulation zu einem bloß logischen Wesen macht. “232 Im Rahmen der zweiten Auflage der Spinoza-Schrift (1789) dient dieses Argument dafür, den in sich widersprüchlichen Begriff einer „Schöpfung aus Ewigkeit her“, mit dem allein der Spinozismus einen Anfangs- und Schöpfungsbegriff denken kann, sowohl zu erklären als auch in seiner Falschheit aufzudecken. Zuvor jedoch, in der Hume-Schrift (1787), hatte Jacobi dasselbe Argument noch im rein systematischen Kontext der Frage nach den Grundformen des außergeistigen Realseins der Dinge zur Anwendung gebracht. Zuerst zeigt Jacobi hier die Bedingung dafür auf, das Ursache-Wirkungs-Verhältnis als eine bloße Bewusstseinsform zu (miss)verstehen: indem nämlich die rein logische, „operative Zeit“ der Synthese von Vorstellungen im Bewusstsein als Gattung begriffen wird, in die sich die reale Naturzeitlichkeit kausaler Abläufe als Art derselben einordnet. Die reine Form zeitlicher Abfolge wird dabei zum tertium comparationis, um die physische Notwendigkeit kausaler Abläufe als Unterart der begrifflichen Notwendigkeit des Grund-Folge-Zusammenhangs zu konzeptualisieren. Kant hat diesen „Fehler“ bspw. im Paragraphen 29 der Prolegomena begangen, wenn er „den Begriff der Ursache als einen zur bloßen Form der Erfahrung notwendig gehörigen Begriff“

232 Jacobi: Über die Lehre des Spinoza, S. 282. 
(Prol. § 29, S. 83), gemäß seiner Deduktion der Kategorien von den Urteilsformen her, aus der „Form eines bedingten Urteils überhaupt, nämlich eine gegebene Erkenntnis als Grund die andere als Folge zu gebrauchen“ (Prol. § 29, S. 82), und damit also aus der Form des logischen Grund-Folge-Zusammenhangs ableitet.

\begin{abstract}
Und so verhält es sich überall, wo wir eine Verknüpfung von Grund und Folge annehmen; wir werden uns nur des Mannichfaltigen in einer Vorstellung bewußt. Weil aber dieses succeßiv geschieht, und eine gewisse Zeit darüber verfließt, so verwechseln wir dieses Werden eines Begriffes mit dem Werden der Dinge selbst, und glauben die würkliche Folge der Dinge eben so erklären zu können, wie sich die ideale Folge der Bestimmungen unserer Begriffe, aus ihrer nothwendigen Verknüpfung in Einer Vorstellung erklären läßt. ${ }^{233}$
\end{abstract}

Die Zeit, die das Bewusstsein braucht, um den rein logischen Zusammenhang von Grund und Folge nacheinander miteinander zu verbinden, als das Nacheinander, mit dem das Bewusstsein die eigentlich logisch gleichzeitigen Elemente von Grund und Folge nur betrachten kann, muss unterschieden werden von der Realität der physischen Zeit („principium generationis“), in der Kausalverhältnisse Gegenstände überhaupt aufeinander einwirken lassen: Denn sonst geschieht laut Jacobi der kantische Fehler, realphysische Kausalwirkung zu einer Form bloßer Bewusstseinszeit zu machen. Dagegen zielt Jacobis Invektive gerade darauf, dieses Verhältnis umzukehren: „Müssen wir dies Succeßive im Denken nicht aus den Organen, aus dem Allmählichen der Bewegung, welcher sie unterworfen sind: folglich aus etwas ausser der Denkkraft erklären?" ${ }^{324}$ Hier ist der ,positive“ Argumentationsansatz Jacobis zur Bestimmung der reinen Form des Realen erreicht: indem er gegen Kant zeigen will, dass die kausale Sukzession als eine erste reine Bestimmung des Realen selbst gedacht werden muss, die in der sinnlichen Erfahrung unmittelbar als etwas außer dem Subjekt, an sich selbst Bestimmtes des Realen auftritt.

Ich habe schon anderwärts dieses Verfahren beleuchtet, und, wie ich glaube, hinlänglich dargetan, daß der Begriff der Ursache, in so fern er sich von dem Begriffe des Grundes unterscheidet, ein Erfahrungsbegriff ist, den wir dem Bewußtsein unserer Kausalität und Passivität zu verdanken haben, und der sich eben so wenig aus dem bloß idealischen Begriffe des Grundes herleiten, als in denselben auflösen läßt. ${ }^{235}$

Diese bewusstseinsexternalistische Kausalbestimmung des Realen selbst erschließt sich jedoch laut Jacobi nur von der Handlungsart, d. h. der Praxisform des

233 Jacobi: David Hume, JWA 2,1, S. 50.

234 Jacobi: David Hume, JWA 2,1, S. 52.

235 Jacobi: Über die Lehre des Spinoza, S. 282. 
menschlichen Weltbezuges. Nur im unmittelbar evidenten Selbstbewusstsein des Ich als Handelnder und der in ihm wirksamen dynamischen Beziehungen von Kraft und Tat ist die Realität der zeitlichen Abfolge dieser Relationen in „Tun“ und „Leiden“ mitgegeben. Denn indem wir das „Gefühl unserer eigenen Kraft“ einzig darin haben, im „Gefühl ihres Gebrauchs [...] einen Widerstand zu überwinden“236, der uns als unabweisbar Anderes entgegentritt, ist die Erfahrung von physischen Verursachungs-Wirkungs-Beziehungen einzig als Überschreitung des Unterschiedes von Subjekt und „Ding an sich“ gegeben. Vom Ich als Handelndem aus erzwingt die Erfahrung physischer Kausalität die Überschreitung der Bewusstseinsgrenze: Ich erfahre Kausalität als etwas meine geistige wie körperliche Außengrenze wirklich Überschreitendes. Bereits die Grundform des Bewusstseins selbst ist so nur in Bezug auf den Einfall eines Anderen, an sich selbst bestimmten und realausgedehnten Daseienden beschreibbar, sodass gilt, „daß zu unserm menschlichen Bewustseyn [...] ausser dem empfindenden Dinge, noch ein würkliches Ding, welches empfunden wird, nothwendig sey. Wir müssen uns von Etwas unterscheiden. Also zwey würkliche Dinge ausser einander, oder Dualität. “237 In einer beinahe naturalistischen Wendung, die Fichtes später entworfene Grunddualität von Ich und Nicht-Ich physikalistisch ausbuchstabiert, werden die Elemente gegenseitiger „Berührung“ in der Beziehung des Ich auf externe Gegenstände, die in ihr erfahrene „Undurchdringlichkeit von beyden Seiten“ sowie der durch sie gegebene „Widerstand im Raume“ als „Würkung und Gegenwürkung“'238 zu den kategorialen Bedingungen von Realität aus der Perspektive des Bewusstseins: aber als Minimalbedingungen von Realsein, welches das Subjekt als nicht durch es selbst konstituierte erfährt. Die Rückführung der realen Sukzession und damit von „Zeitlichkeit überhaupt“ auf die objektiven, transsubjektiven Bedingungen räumlicher Widerständigkeit von Gegenständen aneinander ermöglicht es so, „Grundbegriffe“ in „wahre[r] objective[r] Bedeutung“2339, also kategoriale Bedingungen des „Realseins überhaupt“, zu formulieren: „ die Begriffe [...] von Substanz oder Individualität, von cörperlicher Ausdehnung, von Succeßion, und von Ursache und Wirkung“ müssen ,in den Dingen an sich ihren vom Begriffe unabhängigen Gegenstand, folglich eine wahre objective Bedeutung haben. “240 Wiederum ist es also die unmittelbare Empfindung des Realseins, hier das bestimmte Gefühl räumlich-körperlicher Widerständigkeit, durch welche sich die fundamentalen Bestimmungen von „Realität überhaupt“ dem Subjekt offen-

236 Jacobi: David Hume, JWA 2,1, S. 55.

237 Jacobi: David Hume, JWA 2,1, S. 57.

238 Jacobi: David Hume, JWA 2,1, S. 59.

239 Jacobi: David Hume, JWA 2,1, S. 60.

240 Jacobi: David Hume, JWA 2,1, S. 60. 
baren: „Durch den Glauben wissen wir, daß wir einen Körper haben, und daß außer uns andre Körper und andre denkende Wesen vorhanden sind. [...] denn ohne $D u$, ist das Ich unmöglich.“ ${ }^{241}$ Bei aller Fundamentalkritik am System des Spinoza kommt Jacobi hier mit diesem darin überein, dass das Sein als ,absolute Position“ ${ }^{242} \mathrm{zu}$ verstehen ist (eine Position im Übrigen, die Jacobi nicht nur anhand von Spinozas Substanzbegriff und Kants Kritik des ontologischen Gottesbeweises, sondern auch anhand von Kants vorkritischer Schrift Der einzig mögliche Beweisgrund zu einer Demonstration des Dasein Gottes von 1763 gewonnen hat: 243

Das Sein ist keine Eigenschaft, ist nichts Abgeleitetes von irgend einer Kraft; es ist das, was allen Eigenschaften, Beschaffenheiten und Kräften zum Grunde liegt; das, was man durch das Wort Substanz bezeichnet; wovor nichts kann gesetzt werden, und das Allem vorausgesetzt werden muß.244

Der Intentionalität des Bewusstseins und seinen kategorialen Formen ist so das Bezogensein auf eine an sich bestimmte Realität, die in der sinnlichen Wahrnehmung in das Bewusstsein hineinragt, ohne dass sich das Bewusstsein dabei sinnvoll als Urheber bestimmter Minimalbestimmungen in Form zuschreibbarer Eigenschaften verstehen kann, unaufhebbar eingeprägt. Folglich sind das ,ausser einander“ aller „wahrhaft würkliche[n] Dinge“ und ihr gemeinsamer Zusammenhang in der wechselseitigen äußeren Bestimmung der spinozistisch inspirierte Begriff der endlichen Wirklichkeit, den Jacobi bejaht. ${ }^{245}$ Durch ihn sind die „Begriffe von Einheit und Vielheit, von Thun und Leiden, von Ausdehnung und Succeßion“246 nicht als Formen des Bewusstseins, sondern des Seins zu fassen, als Bedingungszusammenhang des „Realseins überhaupt“, der sich zugleich mit seinem Gegebensein unmittelbar als solcher im „Gefühl“ mitteilt. Die unmittelbare sinnliche Erfahrung des Realen im Gefühl bezeugt dem Ich „ein distinctes reales Medium zwischen Realem und Realem, ein würkliches Mittel von Etwas zu Etwas“ ${ }^{247}$ als „,wesentliche Verhältnisse [...] objectiv realer Bestimmungen.“ ${ }^{248}$ Kants

241 Jacobi: Über die Lehre des Spinoza, S. 114.

242 Förster: Die 25 Jahre der Philosophie, S. 92.

243 Vgl. Förster: Die 25 Jahre der Philosophie, S. $92 \mathrm{f}$.

244 Jacobi: Über die Lehre des Spinoza, S. 65.

245 Vgl. Jacobi: Über die Lehre des Spinoza, S. $263 \mathrm{f}$.: ,[E]in jedes einzelnes Ding [setzt] alle übrige einzelne Dinge voraus, und seine Natur und Beschaffenheit [wird] durch seinen Zusammenhang mit allen übrigen durchaus bestimmt“.

246 Jacobi: David Hume, JWA 2,1, S. 85; vgl. Jacobi: David Hume, JWA 2,1, S. 109.

247 Jacobi: David Hume über den Glauben, S. 109.

248 Jacobi: David Hume über den Glauben, S. 109. 
Begriff der „Sinnlichkeit“ als Medium passiver Rezeptivität wird so folgerichtig nicht als Fakultät des Subjekts, sondern als ein den realen Unterschied des Subjekts und des Gegenstandes außer uns wiederum einschließendes und umfassendes Medium gedacht:

das heißt, daß sowohl die Sinnlichkeit selbst als auch die in diesem Medium aufeinander bezogenen Seiten des Gegenstandes und des affizierten Subjekts als auch die in dieser Beziehung enthaltene Bestimmung der Kausalität in einem emphatischen Sinne als real gekennzeichnet werden müssen. ${ }^{249}$

Damit hat Jacobi eine Lösung für die kantischen Aporien des Begriffs der reinen Realität erarbeitet: indem er nachweist, dass einige der von Kant als rein transzendental, subjektimmanent gedachten Bestimmungsformen - sowohl Zeitlichkeit und Räumlichkeit als Anschauungsformen als auch kategoriale Muster wie Kausalität und Wirksamkeit - auf gleicher Ebene als Elemente des außersubjektiven „Realseins überhaupt“ zu denken sind bzw. den Unterschied von transzendentaler Subjektivität und außersubjektiver Realität umgreifen. Der begriffliche Gehalt von Realsein wird nicht wie bei Kant auf eine bzw. zwei bewusstseinsimmanente Kategorien einzugrenzen gesucht, die dann ihre Explikation, wie im Kant-Kapitel dieser Arbeit gezeigt, überschreiten müssen. Vielmehr anerkennt Jacobi von Anfang an den komplexen, „konkreten“ Charakter des reinen „Realseins überhaupt“ als aus kategorialen Elementen zusammengesetzte Kategorie zweiter Ordnung. Zugleich zeigt Jacobi auf, dass die unendliche Abstraktion des Verstandes von allem Realen, dessen vorgängiges Bestimmtsein bei Kant zu einem gänzlich leeren Begriff wird ${ }^{250}$, auch die Spontaneität des Bewusstseins nicht mehr sinnvoll zu erklären vermag. Damit trifft er eben den konzeptionellen Grundimpuls der Transgressivität des reinen Begriffs der Realität, wie er im Kant-Teil als Problemhorizont der kantischen Philosophie entworfen worden ist: „Jede Bestimmung setzt etwas schon bestimmtes voraus“251, das als immer schon bestimmt, nämlich selbstbestimmt, gedacht werden muss:

Wie sehr nun auch, das Individuum von aussen her bestimmt werden mag, so kann es doch nur zufolge den Gesetzen seiner eigenen Natur bestimmt werden, und bestimmt sich in so fern also selbst. Es muß schlechterdings etwas für sich seyn, weil es sonst nie etwas für ein anderes seyn, und diese oder jene zufällige Bestimmung annehmen könnte. ${ }^{252}$

249 Sandkaulen: Das leidige Ding an sich, S. 186.

250 Vgl. Jacobi: Einleitung, JWA 2,1, S. 404f., $414 \mathrm{f}$.

251 Jacobi: Über die Lehre des Spinoza, S. 167.

252 Jacobi: David Hume, JWA 2,1, S. 56; vgl. auch Jacobi: David Hume, JWA 2,1, S. 77. 
Folglich kann Jacobi die fundamentale, reine Form des Realseins als „Mitdasein“253 bezeichnen. In dieser sind die Extreme bloßer Passivität und bloßer Spontaneität, bloßen Bestimmtwerdens und bloßen Bestimmens verschwunden: und zwar dergestalt, dass sich eine Mehrzahl von „Gegenständen überhaupt“, wollen sie in minimaler kategorialer Beschreibung als ,wirklich' gelten, a) erst dadurch wechselseitig durch je andere bestimmt werden, indem sie an sich selbst und durch sich selbst als immer schon bestimmte vorauszusetzen sind, und b) ihr mit ihrem Bestimmtwerden gleichursprüngliches Immer-schon-Bestimmtsein in der Beziehung auf je andere als ihr Selbstbestimmtsein dem anderen auch prinzipiell offenbar machen, und zwar in der selbstevidenten sinnlichen Wahrnehmbarkeit ihres Dass-Seins und So-Seins: „[D]enn was nicht schon etwas ist, kann nicht zu etwas bloß bestimmt werden; was an sich keine Eigenschaft hat, in dem können durch Verhältnisse keine erzeugt werden, ja es ist nicht einmal ein Verhältnis in Absicht seiner möglich.“254

Jacobis Alternative zu Kants problembehafteten Kategorien von Realität findet ihre Grenze gerade im Umgang mit der Idee der Grenze selbst - der Grenze des Begrifflichen nämlich. Einerseits erwächst seine Philosophie nicht nur aus der Kritik kantischer Subjektivität, sondern auch aus der des spinozistischen physikalischen Naturalismus: weil Spinoza nach Jacobi die Totalität des Wirklichkeitszusammenhangs als vollständig in kausalmechanischen Begriffen zu beschreiben denkt. Die Pointe von Spinozas Rationalismus sieht Jacobi darin, in Ablehnung jeglicher Anfangs- oder Endursachen und damit von Freiheit überhaupt das physikalische Grundgesetz materieller Körper (den reinen UrsacheWirkungs-Zusammenhang) zum Seinsgesetz der Substanz, d.h. alles in ihr im Zusammenhang stehenden Endlichen überhaupt, zu verabsolutieren ${ }^{255}$ und auch alle Erscheinungen der geistigen Welt auf kausalmechanische Begriffe zurückgeführt zu haben. Spinozas physikalischer Monismus begeht dabei für Jacobi den Fehler, aus der Einsicht, „daß sich gewisse Dinge nicht entwickeln lassen“ “256, nämlich in deutlichen Begriffen rekonstruierter Begründungszusammenhänge, die Schlussfolgerung zu ziehen, sie wären überhaupt nicht denkbar und bloße Scheingebilde menschlichen Bewusstseins. Dem setzt Jacobi programmatisch ein reflexives Eingedenken der Grenze des rationalen Prinzips deutlichen Erklärens entgegen, welches das Jenseits dieser Grenze nicht für ungültig und unbestimmbar erklärt, sondern vielmehr als Anderes zur Rationalität bestimmt. ${ }^{257}$

253 Jacobi: Über die Lehre des Spinoza, S. 166.

254 Jacobi: Über die Lehre des Spinoza, S. 172.

255 Jacobi: Über die Lehre des Spinoza, S. 26-34.

256 Jacobi: Über die Lehre des Spinoza, S. 33.

257 Jacobi: Über die Lehre des Spinoza, S. $34 \mathrm{f}$. 
Damit ist es ihm möglich zu betonen, dass es „unmöglich sei, das Unendliche aus dem Endlichen zu entwickeln, und den Übergang des einen zu dem andern“ ${ }^{258}$ nach dem Modell endlichen Werdens des Bedingten aus Bedingungen zu verstehen. Es kann „keine natürliche Philosophie des Übernatürlichen geben“259, in der ein absoluter Grund des Seienden dergestalt mit dem Endlichen zusammenfällt, dass er mit diesem auf endliche Weise - nämlich als Bedingung eines Bedingten - verbunden ist. Der Graben zwischen Unendlichem und Endlichem, den Jacobi des Öfteren als nur durch einen „Sprung“ („Salto mortale“)260 zu überwinden sieht, bestimmt das Unendliche als Übernatürliches: d.h. als nicht in den Begriffen des natürlichen Bedingtseins und Bedingens beschreibbar. ${ }^{261}$ Damit aber gerät die Beziehung der endlichen Wirklichkeit zu ihren letzten, nicht mehr abzuleitenden Gründen - den endlichen wie den unendlichen - zu einem $a b$ strakten, unvermittelten Gegensatz. Ebenso wie Kants Beziehung des Denkens auf das „Ding an sich“, die notwendig unerklärbar und widersprüchlich bleibt, droht Jacobis Beziehung des Denkens auf das „Dasein“ als „das Unauflösliche, Unmittelbare, Einfache“262 die Gefahr, nicht sinnvoll beschreibbar zu sein und in der bloß irrationalen Mystik einer unmittelbaren Offenbarung zu verschwinden.

All diese Schwierigkeiten - und die Grenze des jacobischen Transgressivitätskonzepts überhaupt - gründen in dem zu engen Begriff des Begriffs, den Jacobi von Spinoza übernimmt: zu eng zum einen in der Rückführung der begrifflichen Funktion auf die Repräsentation (Form des intentionalen Gehalts) wie Nachbildung (Form des Begriffs selbst) natürlicher Kausalität; zu eng zum anderen in der Restriktion der begrifflichen Funktion auf verständesmäßige Bewusstseinsvollzüge von Subjekten. Zwar kritisiert Jacobi an Spinoza eine Naturalisierung der Wirklichkeit insgesamt; zugleich aber übernimmt und verschärft er die Naturalisierung des Begrifflichen, die er bei Spinoza verortet hat. „[S]o bleiben wir, so lange wir begreifen, in einer Kette bedingter Bedingungen. Wo diese Kette aufhört, da hören wir auf zu begreifen, und da hört auch der Zusammenhang, den wir Natur nennen, selbst auf.“263 Die „Konstruktion eines Begriffes überhaupt“ als „a priori aller Konstruktionen“264 besteht darin, „eine Sache [...] aus ihren nächsten Ursprüngen herleiten [zu] können“265. Diese „progressive Verknüpfung“ nach den

258 Jacobi: Über die Lehre des Spinoza, S. 37.

259 Jacobi: Über die Lehre des Spinoza, S. 271.

260 Jacobi: Über die Lehre des Spinoza, S. 26.

261 Jacobi: Über die Lehre des Spinoza, S. 288.

262 Jacobi: Über die Lehre des Spinoza, S. 35.

263 Jacobi: Über die Lehre des Spinoza, S. 288.

264 Jacobi: Über die Lehre des Spinoza, S. 285.

265 Jacobi: Über die Lehre des Spinoza, S. 284. 
„Gesetzen der Notwendigkeit, das ist, des Identischen“266 legt die Funktion und Struktur des Begriffs auf die deutliche Repräsentation kausaler Beziehungen als Gefüge der Bedingungen der Genese einer Sache fest. Der „Mechanismus des Prinzips“267 des Begriffs, nach dem wir „keine Begriffe, als Begriff des bloß Natürlichen zu bilden im Stande sind“268, ist das „Kausalitätprinzip des Verstandes“269 selbst. Damit aber gerät die selbst begriffliche Erklärung des Unterschiedes, den Jacobi zwischen dem Natürlichen und dem Übernatürlichen ansetzt, in Widerspruch zu sich selbst: Erklärt wird ein Unterschied, bei dem ein Glied des Unterschiedes in einem absoluten Jenseits der Möglichkeiten des begrifflichen Erklärens liegt; beschrieben wird so eine Schranke als Grenze. Hier verliert sich Jacobis reflexives Eingedenken der Widersprüche der kantischen Philosophie in eben den Schwierigkeiten, die er zu markieren sucht. Die Transgressivität des reinen Begriffs des Realen, d.h. das Hineinragen eines vorgängig an sich selbst bestimmten Realen in seine Bestimmbarkeit durch das Bewusstsein, kann nicht mit der Grenze des Begrifflichen zu einem vollständig Nichtbegrifflichen zusammenfallen. So wird deutlich, dass es eines erweiterten, komplexeren und differenzierteren Begriffs des Begrifflichen bedarf, um die Transgressivität des reinen Begriffs des Realen angemessen und nicht-widersprüchlich denken zu können. Einen Ansatz dafür gibt es bei Jacobi dort, wo er die Vernunft nicht bloß als Seelenvermögen des Menschen versteht, sondern als eine transsubjektive geistige Form des Wirklichen überhaupt. ${ }^{270}$ Hegel wird eben diesen Weg weiter beschreiten.

266 Jacobi: Über die Lehre des Spinoza, S. 285.

267 Jacobi: Über die Lehre des Spinoza, S. 288.

268 Jacobi: Über die Lehre des Spinoza, S. 291.

269 Jacobi: Über die Lehre des Spinoza, S. 311.

270 Jacobi: Über die Lehre des Spinoza, S. 86. 